The Astrophysical Journal, 685:1320-1323, 2008 October 1

(C) 2008. The American Astronomical Society. All rights reserved. Printed in U.S.A.

\title{
ERRATUM: "GROUPS OF GALAXIES IN THE TWO MICRON \\ ALL SKY REDSHIFT SURVEY” (ApJ, 655, 790 [2007])
}

\author{
Aidan C. Crook, John P. Huchra, Nathalie Martimbeau, \\ Karen L. Masters, Tom Jarrett and Lucas M. Macri
}

A computational error resulted in a scaling of the linking parameter, $D$, with a weaker dependence on redshift than that described in the text. Figure E1 shows linking length $D(V)$ as a function of redshift, $V$, in both utilized and corrected forms. The mean intergalaxy separation (of visible galaxies) computed from the luminosity function for a $K<11.25$ survey is also shown, for scale.

Since the redshift distribution of visible galaxies peaks near $6000 \mathrm{~km} \mathrm{~s}^{-1}$, where the difference between the scalings is minimized, the overall properties of the groups are consistent (within reported $99 \%$ confidence levels) with the values in the original article. The revised values are given here in the corrected Table 2 ; the conclusions of the article remain unchanged. The change in group identification is most significant very nearby $\left(V \lesssim 1500 \mathrm{~km} \mathrm{~s}^{-1}\right)$, where the reduced linking length results in fewer groups merging with the Virgo group.

The values of $D_{0}=D\left(V_{F}=1000 \mathrm{~km} \mathrm{~s}^{-1}\right)$ should read $1.04 \mathrm{Mpc}$ for the LDC catalog $(\delta \rho / \rho=12)$ and $0.56 \mathrm{Mpc}$ for the HDC catalog $(\delta \rho / \rho=80)$. The linking parameters obtained when maximizing the number of groups using the corrected algorithm are consistent with those selected for the definition of the LDC catalog. We present the revised LDC and HDC group catalogs, at density contrasts $\delta \rho / \rho=$ 12 (setting $V_{0}=399 \mathrm{~km} \mathrm{~s}^{-1}$ ) and $\delta \rho / \rho=80$ (setting $V_{0}=350 \mathrm{~km} \mathrm{~s}^{-1}$ ), respectively. The full corrected catalogs (Tables 6, 7, 10, and 11) are available in electronic form with the online materials accompanying this erratum. We are grateful to Guilhem Lavaux for bringing the error to the attention of the authors.

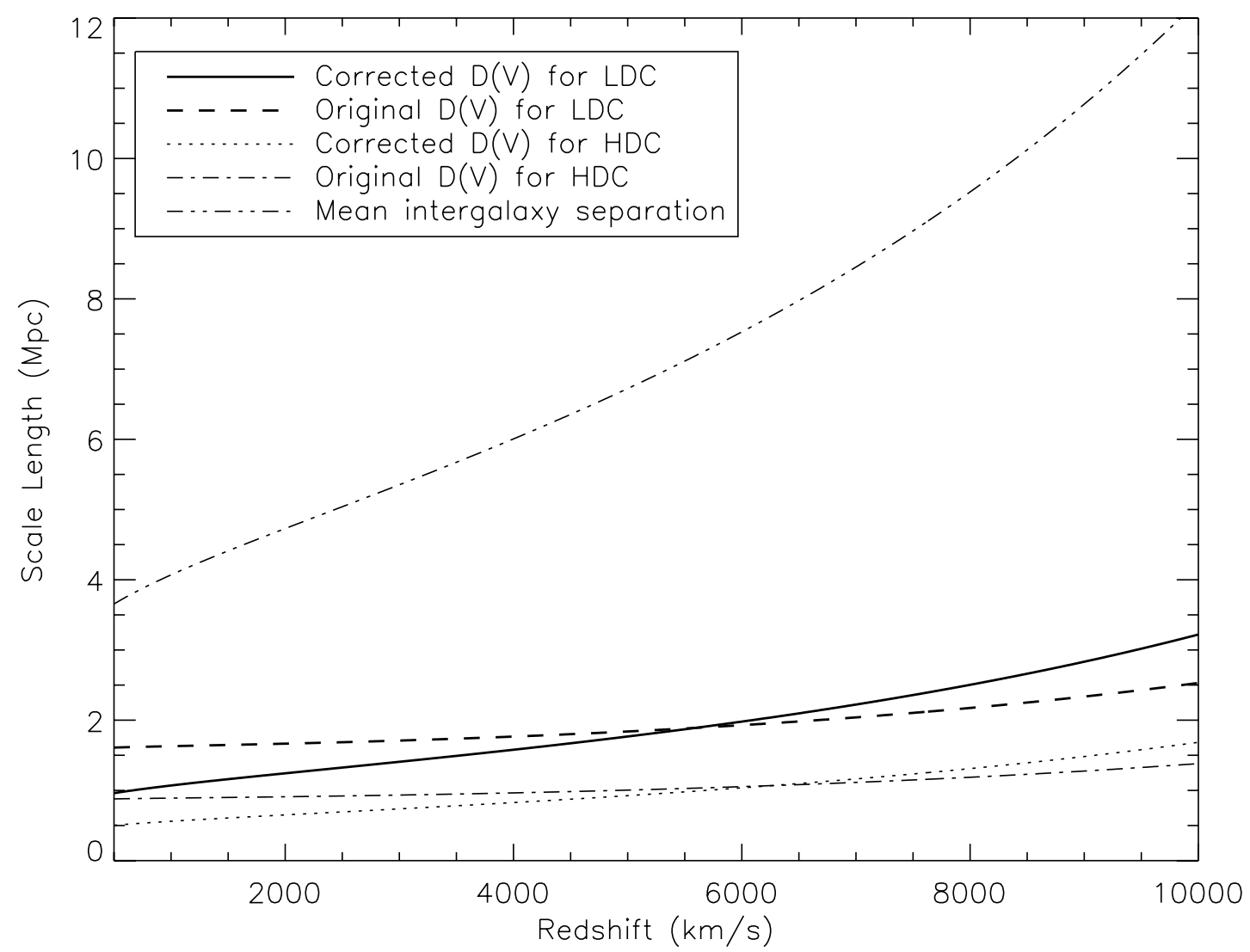

FIG. E1.- Linking parameter as a function of redshift for the density contrasts corresponding to the LDC and HDC catalogs. The original and corrected values are plotted, along with the mean intergalaxy separation. We assume $h=0.73$ where a value is required. 
TABLE 2

Median Properties of Groups with Five or More Genuine Members

\begin{tabular}{|c|c|c|}
\hline Property & LDC Catalog & HDC Catalog \\
\hline$\sigma_{P}\left(\mathrm{~km} \mathrm{~s}^{-1}\right)$ & $196(184,205)$ & $187(175,202)$ \\
\hline$R_{\mathrm{PV}}(\mathrm{Mpc}) \ldots \ldots$ & $1.67(1.56,1.79)$ & $0.89(0.81,0.96)$ \\
\hline $\log \left(M_{V} / M_{\odot}\right)$ & $13.82(13.74,13.90)$ & $13.49(13.42,13.58)$ \\
\hline $\log \left(M_{P} / M_{\odot}\right) \ldots \ldots \ldots$ & $14.05(13.98,14.11)$ & $13.63(13.57,13.71)$ \\
\hline $\log \left[\left(M_{V} / L_{K}\right) /\left(M_{\odot} / L_{\odot}\right)\right] \ldots \ldots \ldots \ldots \ldots \ldots \ldots$ & $1.70(1.64,1.75)$ & $1.52(1.45,1.58)$ \\
\hline $\log \left[\left(M_{P} / L_{K}\right) /\left(M_{\odot} / L_{\odot}\right)\right] \ldots \ldots \ldots \ldots \ldots \ldots \ldots$ & $1.90(1.83,1.95)$ & $1.64(1.57,1.70)$ \\
\hline$\Omega_{M, V}$ & $0.14(0.12,0.16)$ & $0.09(0.08,0.11)$ \\
\hline$\Omega_{M, P}$ & $0.22\left(\begin{array}{ll}0.19 & 0.25)\end{array}\right.$ & $0.13(0.11,0.14)$ \\
\hline
\end{tabular}

TABLE 6

LDC Catalog of Groups in the 2MASS Redshift Survey

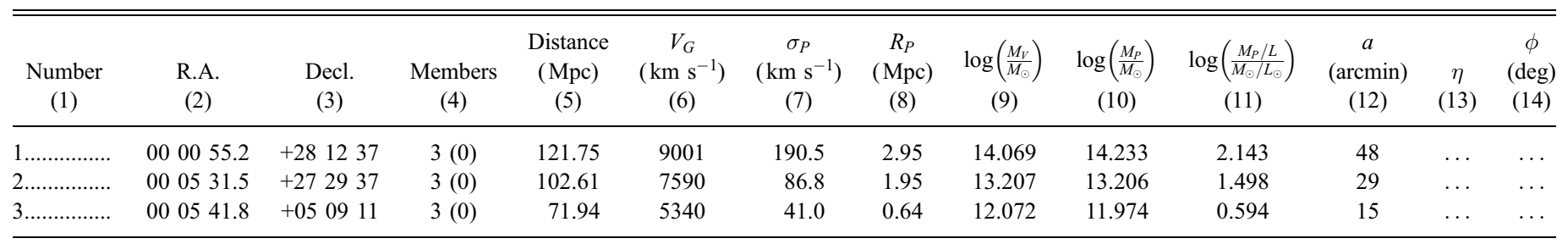

Notes. - This catalog has been produced using parameters $\left(D_{0}, V_{0}\right)=\left(1.04 \mathrm{Mpc}, 399 \mathrm{~km} \mathrm{~s}^{-1}\right)$, corresponding to the density contrast $\delta \rho / \rho=12$. We assume $h=$ 0.73 where a value is required. Col. (4): Number of group members (including those generated from the population of the plane). The number derived from the Galacticplane population is contained in parentheses. Col. (5): Mean (corrected) group distance. Col. (6): Mean heliocentric group velocity. Col. (7): Line-of-sight velocity dispersion. Col. (8): Projected virial radius. Col. (9): Log of the virial mass in solar units. Col. (10): Log of the projected mass in solar units. Col. (11): Log of the (projected) mass-to-light ratio in solar units. Col. (12): Semimajor axis of the ellipse fit to the group at the 75th percentile level. Col. (13): Axis ratio of ellipse fit to the group members. Col. (14): Position angle of semimajor axis of ellipse fit to the group members; measured from north toward east. Table 6 is published in its entirety in the electronic edition of the Astrophysical Journal. A portion is shown here for guidance regarding its form and content. 
TABLE 7

hDC Catalog of Groups in the 2MASS Redshift Survey

\begin{tabular}{|c|c|c|c|c|c|c|c|c|c|c|c|c|c|c|}
\hline $\begin{array}{l}\text { Number } \\
\text { (1) }\end{array}$ & $\begin{array}{l}\text { R.A. } \\
\text { (2) }\end{array}$ & $\begin{array}{l}\text { Decl. } \\
\text { (3) }\end{array}$ & $\begin{array}{c}\text { Members } \\
\text { (4) }\end{array}$ & $\begin{array}{l}\text { Distance } \\
\text { (Mpc) } \\
\text { (5) }\end{array}$ & $\begin{array}{c}V_{G} \\
\left(\mathrm{~km} \mathrm{~s}^{-1}\right) \\
(6)\end{array}$ & $\begin{array}{c}\sigma_{P} \\
\left(\mathrm{~km} \mathrm{~s}^{-1}\right) \\
(7)\end{array}$ & $\begin{array}{c}R_{P} \\
(\mathrm{Mpc}) \\
(8)\end{array}$ & $\begin{array}{c}\log \left(\frac{M_{V}}{M_{\odot}}\right) \\
(9)\end{array}$ & $\begin{array}{c}\log \left(\frac{M_{P}}{M_{\odot}}\right) \\
(10)\end{array}$ & $\begin{array}{c}\log \left(\frac{M_{P} / L}{M_{\odot} / L_{\odot}}\right) \\
(11)\end{array}$ & $\begin{array}{c}a \\
(\operatorname{arcmin}) \\
(12)\end{array}$ & $\begin{array}{c}\eta \\
(13)\end{array}$ & $\begin{array}{c}\phi \\
(\mathrm{deg}) \\
(14)\end{array}$ & $\begin{array}{c}\text { Corresponding } \\
\text { Group Number } \\
\text { (15) }\end{array}$ \\
\hline 1 & 000531.5 & +272937 & $3(0)$ & 102.61 & 7590 & 86.8 & 1.95 & 13.207 & 13.206 & 1.498 & 29 & & & 2 \\
\hline 2 & 000541.8 & +050911 & $3(0)$ & 71.94 & 5340 & 41.0 & 0.64 & 12.072 & 11.974 & 0.594 & 15 & & & 3 \\
\hline 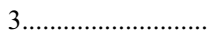 & 000610.8 & +470059 & $4(0)$ & 70.80 & 5162 & 158.1 & 0.56 & 13.183 & 13.544 & 1.970 & 33 & & & 6 \\
\hline
\end{tabular}

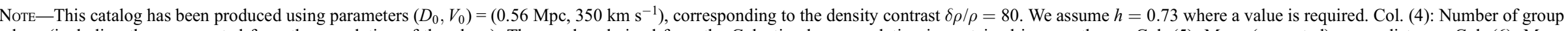

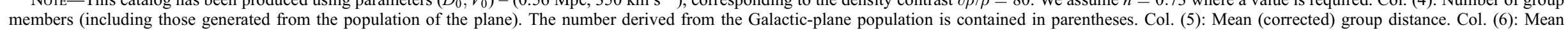

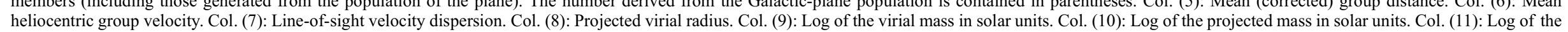

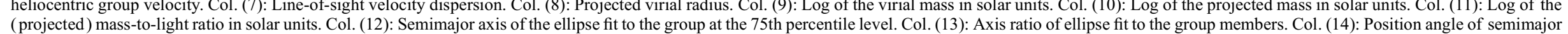

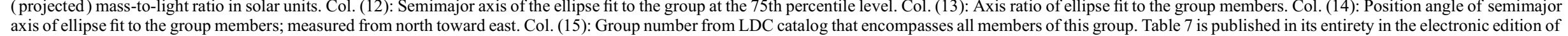
axis of ellipse fit to the group members; measured from north toward east. Col. (15): Group number
the Astrophysical Journal. A portion is shown here for guidance regarding its form and content. 
TABLE 10

Groups in the LDC Catalog and Their Members

\begin{tabular}{|c|c|c|c|c|c|c|}
\hline $\begin{array}{l}\text { Name } \\
\text { (1) }\end{array}$ & $\begin{array}{l}\text { R.A. } \\
\text { (2) }\end{array}$ & $\begin{array}{c}\text { Decl. } \\
\text { (3) }\end{array}$ & $\begin{array}{c}V_{h} \\
\left(\mathrm{~km} \mathrm{~s}^{-1}\right) \\
(4)\end{array}$ & $\begin{array}{l}m_{K} \\
(5)\end{array}$ & $\begin{array}{c}\text { Distance } \\
(\mathrm{Mpc}) \\
(6)\end{array}$ & $\begin{array}{l}\text { Corresponding } \\
\text { Group Number } \\
\text { (7) }\end{array}$ \\
\hline \multicolumn{7}{|c|}{ Group 1} \\
\hline 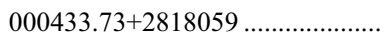 & 000433.6 & +281806 & 8785 & 10.62 & 118.79 & None \\
\hline $235828.41+2802025 \ldots \ldots \ldots \ldots \ldots \ldots$ & 235828.3 & +280203 & 9145 & 10.94 & 123.72 & None \\
\hline 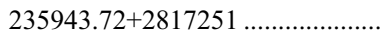 & 235943.6 & +281725 & 9073 & 10.71 & 122.74 & None \\
\hline \multicolumn{7}{|c|}{ Group 2} \\
\hline $000329.22+2721063 \ldots \ldots \ldots \ldots \ldots \ldots$ & 000329.1 & +272106 & 7690 & 11.02 & 103.97 & 1 \\
\hline $000548.43+2726579 \ldots \ldots \ldots \ldots \ldots \ldots$ & 000548.3 & +272658 & 7531 & 10.95 & 101.81 & 1 \\
\hline $000717.10+2740421 \ldots \ldots \ldots \ldots \ldots \ldots \ldots$ & 000717.1 & +274042 & 7550 & 11.13 & 102.05 & 1 \\
\hline
\end{tabular}

NoтE.-The LDC group catalog was created using $\left(D_{0}, V_{0}\right)=\left(1.04 \mathrm{Mpc}, 399 \mathrm{~km} \mathrm{~s}^{-1}\right)$, corresponding to a density contrast $\delta \rho / \rho=12$. Col. (4): Heliocentric velocity. Col. (5): Corrected distance, assuming $h=0.73$. Col. (6): Apparent $K$ magnitude. Col. (7): Corresponding group number assigned to this galaxy in the HDC catalog. Table 10 is published in its entirety in the electronic edition of the Astrophysical Journal. A portion is shown here for guidance regarding its form and content.

TABLE 11

Groups in the HDC Catalog and Their Members

\begin{tabular}{|c|c|c|c|c|c|c|}
\hline $\begin{array}{l}\text { Name } \\
\text { (1) }\end{array}$ & $\begin{array}{l}\text { R.A. } \\
\text { (2) }\end{array}$ & $\begin{array}{c}\text { Decl. } \\
\text { (3) }\end{array}$ & $\begin{array}{c}V_{h} \\
\left(\mathrm{~km} \mathrm{~s}^{-1}\right) \\
(4)\end{array}$ & $\begin{array}{l}m_{K} \\
(5)\end{array}$ & $\begin{array}{c}\text { Distance } \\
(\mathrm{Mpc}) \\
(6)\end{array}$ & $\begin{array}{c}\text { Corresponding } \\
\text { Group Number } \\
\text { (7) }\end{array}$ \\
\hline \multicolumn{7}{|c|}{ Group 1} \\
\hline 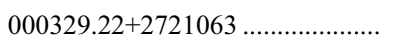 & 000329.1 & +272106 & 7690 & 11.02 & 103.97 & 2 \\
\hline 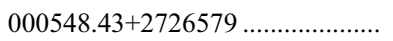 & 000548.3 & +272658 & 7531 & 10.95 & 101.81 & 2 \\
\hline 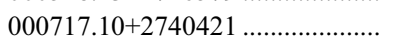 & $0007 \quad 17.1$ & +274042 & 7550 & 11.13 & 102.05 & 2 \\
\hline \multicolumn{7}{|c|}{ Group 2} \\
\hline 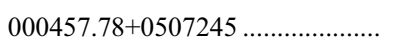 & 000457.8 & +050724 & 5357 & 11.19 & 72.17 & 3 \\
\hline $000527.66+0513204 \ldots \ldots \ldots \ldots \ldots \ldots$ & 000527.6 & +051320 & 5294 & 10.08 & 71.31 & 3 \\
\hline $000640.35+0506483 \ldots \ldots \ldots \ldots \ldots \ldots$ & 000640.3 & +050648 & 5371 & 11.22 & 72.34 & 3 \\
\hline
\end{tabular}

NotE.-The HDC group catalog was created using $\left(D_{0}, V_{0}\right)=\left(0.56 \mathrm{Mpc}, 350 \mathrm{~km} \mathrm{~s}^{-1}\right)$, corresponding to a density contrast $\delta \rho / \rho=80$. Col. (4): Heliocentric velocity. Col. (5): Corrected distance, assuming $h=0.73$. Col. (6): Apparent $K$ magnitude. Col. (7): Corresponding group number assigned to this galaxy in the LDC catalog. Table 11 is published in its entirety in the electronic edition of the Astrophysical Journal. A portion is shown here for guidance regarding its form and content. 